\title{
Original
}

\section{Estenosis uretral en la mujer: Etiologia, diagnóstico y tratamiento}

\author{
Cristina Gutiérrez Ruiz, Fernando Rodríguez-Escovar, Carlos Errando Smet, Pedro Arañó Bertrán, \\ Humberto Villavicencio Mavrich
}

Servicio de Urología. Fundació Puigvert. Barcelona, España

\begin{abstract}
Resumen
La estenosis uretral en la mujer es una causa poco frecuente de obstrucción infravesical, entidad ya de por sí poco habitual en el sexo femenino. La mayoría de los casos son secundarios a procedimientos quirúrgicos del área urogenital, la fibrosis periuretral es el mecanismo de acción implicado independientemente de la causa. Existe cierta controversia en cuanto a los criterios diagnósticos pero parece claro que se trata de obstrucciones estructurales que afectan al tercio medio y distal de la uretra. No existe un consenso en cuanto al tratamiento, pero maniobras menos agresivas como las dilataciones o la uretrotomia interna son las técnicas de elección para las estenosis primarias, teniendo en cuenta el alto porcentaje de recidivas que presentan. Las técnicas de reconstrucción uretral mediante el uso de diversos tipos de injertos o flaps estarían indicadas en aquellos casos recidivados o bien en los que existe un defecto parcial o total uretral.
\end{abstract}

Palabras clave: Uretra femenina. Estenosis. Tratamiento. Uretroplastia.

Female Urethral Stricture: Etiology, diagnostic and treatment

Abstract

Female urethral stricture is an infrequent cause of bladder outlet obstruction, which is also a rare clinical entity in women. The most frequent etiology is the previous urogenital surgery. Periurethral fibrosis is the final cause regardless the etiology. Considerably controversie sorrounds in the diagnostic criteria but the estrictures are structural obstructions which involve media and distal third of the female urethra. There is no treatment consensus, but less agresive maneuvers such as dilatations and urethrotomies are chosen to treat primary estrictures, although the high recurrence rates. Reconstructive tecniques with the use of several flaps and grafts should be considered in recurrent cases and when there is a partial or total urethral defect.

Keywords: Female urethra. Stricture. Treatment. Urethroplasty.

$\mathrm{L}^{2}$ a obstrucción infravesical en la mujer es una entidad poco frecuente en el 3\% y el $8 \%$ de todas las pacientes con clínica de tramo urinario inferior. De éstas, en un 13\% se logra demostrar la presencia de una estenosis uretral mediante uretrocistografía y estudios de Presión-Flujo ${ }^{1}$. Su incidencia es claramente menor que en el hombre, ya sea por su menor longitud, su mayor movilidad o por presentar menor protección por parte del hueso pélvico en toda su longitud ${ }^{2}$.

Existe escasa bibliografía sobre su causa, diagnóstico y tratamiento; y muchos artículos se centran en las patologias pediátricas. Parece que el tratamiento para las estenosis primarias en mujeres adultas es la dilatación uretral o la uretrotomía endoscópica, pero éstas presenta una alta tasa de recidivas $^{3}$, existiendo cierta controversia sobre cuál es la técnica de elección en estenosis recidivadas.
El objetivo del presente artículo es describir la etiología, el diagnóstico y el tratamiento de la estenosis uretral femenina, haciendo hincapié en el tratamiento de las estenosis recidivadas o complejas.

\section{Consideraciones anatómicas}

Tal y como veremos más adelante, es imprescindible conocer la anatomía uretral y sus relaciones, no sólo para el tratamiento de las estenosis sino también a la hora de evitar posibles lesiones uretrales durante cirugías del área pélvica.

Karam et al. realizan un estudio mediante reconstrucción 3D tras tinción histoquímica de las fibras musculares y nerviosas del esfinter y la uretra femenina en fetos humanos ${ }^{4}$. Dicho estudio confirma que el tercio proximal está formado por un esfínter circular de musculatura lisa, el tercio medio 
por 2 capas circulares de musculatura lisa y estriada, y el tercio distal por una capa circular de musculatura lisa rodeado por una capa de músculo estriado en forma de omega que se abre por la parte posterior. En cuanto a la inervación de dichas estructuras, en el tercio proximal identificaron fibras mielínicas junto con amielínicas del plexo pélvico, íntimamente relacionadas con la pared anterior y lateral de la vagina. Identificaron la entrada de las fibras amielínicas al músculo liso esfinteriano a las 4 y $8 \mathrm{~h}$ y la mayoría de fibras amielínicas entraban en el esfinter a las 3 y 9 h.

La musculatura uretral va disminuyendo de grosor a medida que se hace más distal incorporando fibras de tejido conectivo hasta desaparecer. Desde ese punto hasta el meato, la uretra ya no es una estructura muscular sino conectiva con fibras elásticas interpuestas. Un exceso de este tejido colágeno resultaría en una estrechez y disminución de la elasticidad de este segmento uretral; este hallazgo histológico parece ser el causante de los anillos uretrales estenóticos que se encuentran en niñas con infecciones urinarias de repetición ${ }^{5}$.

La compleja estructura de la uretra femenina implica una inervación mixta (autonómica para la musculatura lisa, somática para la musculatura estriada y autonómica y sensorial para la mucosa y la submucosa). Las fibras musculares lisas y estriadas están intimamente relacionadas dificultando la identificación de las fibras nerviosas que inervan cada estructura muscular ${ }^{6}$.

Otra de las consideraciones a tener en cuenta es la anatomía e inervación del esfínter urinario para así evitar lesionarlo durante intervenciones quirúrgicas próximas a éste. Arango ${ }^{7}$ confirmó la existencia de fibras somáticas intrapélvicas que compartían la inervación del esfínter estriado con otras fibras autonómicas derivadas del plexo pélvico en modelos fetales. Por otra parte Stein $^{8}$ afirma que la membrana perineal consta de una porción posterior y otra anterior, esta última es un complejo que engloba el compresor uretral y el esfinter uretrovaginal de la uretra distal y se continúa con el tendón conjunto del periné. Por tanto la disección proximal de la uretra, tanto por la cara ventral como posterior, conlleva un potencial riesgo de lesión del complejo esfinteriano.

Por último, antes de plantear el abordaje quirúrgico nos hemos de plantear las relaciones de la uretra con el cuerpo del clítoris. El tejido eréctil del clí- toris rodea por la cara posterior a la uretra creando el complejo uretrovaginal y dándole soporte. Los cuerpos bulbares del clítoris progresan anteriormente a la uretra hasta unirse en la parte ventral ${ }^{9}$.

\section{Etiologia de la estenosis uretral en la mujer}

La etiología de la estenosis uretral femenina en la mayoría de los casos es desconocida. En países en vías de desarrollo la causa más frecuente es la lesión del tracto urinario durante los trabajos de parto prolongados. En países industrializados las causas más frecuentes son: postraumáticas, lesiones yatrógenas (durante diverticulectomias uretrales, cirugias antiincontinencia, endoscópicas y ginecológicas...), radioterapia pélvica o uretritis agudas y crónicas ${ }^{10}$. Otros factores asociados son la atrofia vulvar en mujeres posmenopáusicas ${ }^{11}$.

Teniendo en cuenta la alta incidencia de instrumentación uretral en la población sana parece que aunque no constituya una causa directa probablemente sea un factor de riesgo para la aparición de estenosis $^{12}$. Edwards et al. describen un modelo animal para valorar los efectos de los diferentes materiales de los catéteres y su diámetro en la mucosa uretral. Estos autores encontraron que cualquier material induce inflamación de la mucosa (siendo la silicona la que menor grado de inflamación produce), los catéteres de menor tamaño también inducen menos inflamación porque permiten la salida del exudado uretral; al mantener más días el catéter uretral se añade el riesgo de colonización por bacterias aumentando también el daño de la mucosa uretral. Estos autores recomiendan el uso de catéteres de silicona de menor diámetro posible y su introducción cuidadosa así como mantenerlos con fijación proximal ${ }^{13}$.

Sea cual sea el origen de la estenosis parece claro que el mecanismo fisiopatológico de la estrechez uretral es la fibrosis periuretral ${ }^{10}$.

\section{DIAGNÓSTICo}

A pesar de que la disfunción de vaciado en la mujer es una entidad frecuente, la obstrucción infravesical es relativamente rara, afectando entre el 3-8\% de estas pacientes, de éstas sólo un 13\% presentaran una estenosis uretral $^{1}$. Existe además cierta controversia entre los autores a la hora de diagnosticarla y no existe una definición clara sobre los criterios que han de usarse para llegar a su diagnóstico. 
Podemos clasificar la obstrucción infravesical en la mujer en funcionales o estructurales (anatómicas); entre las causas estructurales éstas pueden ser intraluminales (litiasis, tumoraciones...) o uretrales como: estenosis de meato, carúnculas, fibrosis, divertículos uretrales y la estenosis uretral ${ }^{14}$.

Algunos autores afirman que sólo las obstrucciones infravesicales distales en la mujer son estructurales, mientras que las obstrucciones proximales son predominantemente funcionales causadas por una inadecuada relajación de la musculatura del suelo pélvico. Las obstrucciones estructurales proximales serian causadas por procesos extrauretrales la mayoría de ellos secundarias a procesos antiincontinencia previos ${ }^{8}$.

En la mujer, definimos estenosis uretral como aquella estrechez fija anatómica que no permite el paso de instrumental sin disrupción de su mucosa. Debe diferenciarse de las estrecheces funcionales o fisiológicas que se evidencian en las exploraciones radiográficas pero que permiten el paso de instrumentales sin disrupción de la luz uretral (p.e. micción disfuncional o disinergia vesico-esfinteriana) ${ }^{11}$.

En ocasiones el fracaso terapéutico de síndromes uretrales y miccionales de vaciado mediante dilataciones uretrales o tomías es debido a un error en el diagnóstico. No existe evidencia clínica de la eficacia de dichas terapias en estos síndromes aumentando el riesgo de desarrollar a posteriori una estenosis anatómica por sucesivas manipulaciones.

Tampoco existe un claro consenso sobre cuál es el diámetro a partir del cual hemos de considerar una uretra femenina como estenótica. Brannan sugiere que una estrechez uretral $\leq 20 \mathrm{~F}$ es patológica y tributaria de tratamiento ${ }^{15}$. Una revisión de la literatura concluye que una uretra femenina adulta que no admita un catéter de $14 \mathrm{Ch}$ es altamente sugestiva de ser obstructiva y que va a condicionar disfunción de vaciado ${ }^{9}$.

\section{RESEÑA HISTÓRICA}

La estenosis uretral en la mujer fue descrita por primera vez por Lisfranc en 1824 y fue Newman el primero en abogar por su importancia y la necesidad de su tratamiento mediante electrolisis. Scott ${ }^{16}$ en 1924 realiza una amplia revisión de lo publicado hasta el momento añadiendo aportaciones propias que justifica con casos clínicos. En dicho trabajo se concluye que la estenosis uretral en la mujer es una patología frecuente, siendo las etiologías principales la uretritis gonocócica y los traumas durante el parto; la localización más frecuente es la uretra distal y el meato. En cuanto al diagnóstico, éste se realiza mediante calibración con sondas y/o bujías y el tratamiento es la dilatación progresiva evitando al máximo la cirugía. En gran medida la literatura clásica se basa en reportes de casos clínicos. Brannan en $1951^{15}$ realiza una nueva revisión con conclusiones similares a las de Scott, coincidiendo con éste en que el tratamiento de elección son las dilataciones progresivas. Lyon ${ }^{17}$ correlaciona el tratamiento mediante calibración-dilatación uretral con la mejoría en los parámetros miccionales en aquellas pacientes con estenosis de uretra medio-distal, y plantea la calibración uretral con flujometria pre y post como herramienta para determinar aquellos casos que van a responder al tratamiento y aquellas que serán tributarias de más estudios por posible obstrucción a nivel proximal.

\section{Tratamiento de la estenosis uretral primaria en la mujer}

El objetivo del tratamiento de la estenosis de uretra es corregir la obstrucción anatómica y funcional, con preservación de la continencia urinaria ${ }^{9}$.

Previamente a la cirugía de la estenosis debe evaluarse cuidadosamente a la paciente con el objeto de descartar patologías concomitantes como la presencia de fístulas vesicovaginales, reflujo vesicouretral, insuficiencia esfinteriana o hiperactividad del detrusor. A pesar de que existe hiperactividad del detrusor o hipoacomodación vesical preoperatoria, dichas condiciones a menudo desaparecerán al corregir la obstrucción por ser secundarias a ésta.

El tratamiento de la estenosis uretral en la mujer dependerá de:

- La localización y longitud de la estenosis.

- La longitud de la uretra sana proximal.

- La integridad o no del cuello vesical.

- La coexistencia de incontinencia.

El método más simple para el tratamiento inicial de la estenosis uretral en la mujer es la dilatación uretral progresiva. Se ha demostrado la eficacia de dicha maniobra en el tratamiento de la estenosis uretral con mejoría perdurable tanto en el diámetro uretral (medido por calibración mediante sondas) como del flujo medio así como una disminución en la presión máxima de cierre uretral ${ }^{18}$. A pesar de considerarse una maniobra sencilla no está exenta de riesgos y la tasa de recurrencias no es desdeñable. 
La dilatación uretral poco cuidadosa conlleva cierto grado de uretrorragia y extravasado lo que podría conducir a fibrosis posterior y recurrencia de la estenosis ${ }^{8}$.

En el momento de valorar la eficacia real de las dilataciones en el tratamiento de la estenosis uretral existe el inconveniente de que hasta un 61\% de los urólogos utilizan la dilatación uretral como tratamiento del sd uretral, sin confirmar la presencia de estenosis; este hecho hace que sea difícil valorar los resultados de la técnica y aumenta las estenosis por dilataciones poco cuidadosas de síndromes uretrales no estenóticos. Estudios recientes demuestran que en los últimos años cada vez menos urólogos la utilizan y la encuentran menos efectiva ${ }^{20}$.

Smith ${ }^{12}$ publica una serie de 7 pacientes, tratadas entre 1999 y 2004, mediante autocateterismos tras dilatación uretral bajo sedación. El diagnóstico se realizó mediante VideoUrodinamia. Realizan una dilatación uretral hasta $30 \mathrm{~F}$ con sondas progresivas y una uretroscopia posterior donde el hallazgo de disrupción de la mucosa se consideró un signo altamente sugestivo de la presencia previa de la estenosis. Recogen biopsias de la estenosis para descartar malignidad y se excluyen pacientes con radioterapia previa. Se dejó catéter Foley durante 1-7d, al retirarlo las pacientes inician el aprendizaje de autocateterismos con sonda 18-20F (al menos 1/día). Si durante el seguimiento la estenosis recidivó, se repitió el procedimiento. Tres de las pacientes necesitaron dilataciones repetidas y una paciente requirió uretrotomia fría con posteriores autocateterismos, dicha paciente inicialmente no había cumplido con el programa de autocateterismos.

Debido al riesgo de recurrencia, al dejar los autocateterismos, estos pacientes permanecieron en autocateterismos durante largos períodos. Todas las pacientes presentaron mejoras objetivas con la dilatación uretral, ninguna presentó incontinencia ni complicaciones. Aparentemente el cumplimiento con los autocateterismos fue el único factor asociado a la recidiva, por lo que el trabajo concluye que aquellas mujeres que son poco cumplidoras o con poca destreza manual, deberían tratarse inicialmente con una cirugía reconstructiva.

Si valoramos la tasa de éxitos global de dicha práctica (pacientes que han sido solventadas con una sola dilatación y autocateterismos posteriores) vemos que ésta es tan sólo del $42 \%$, lo que confirma la alta recurrencia de estenosis postdilatación ya comentada previamente.
Para pacientes seleccionadas con estenosis cortas la uretrotomía interna sería una opción correcta, pero en estenosis proximales existe el riesgo de producir una lesión esfinteriana con la consecuente incontinencia urinaria ${ }^{10}$. Un requisito indispensable para su realización es que la luz de la uretra indemne sea lo suficientemente amplia para permitir el paso del instrumental endoscópico. Las ventajas de dicha técnica son la escasa morbilidad y la estancia hospitalaria corta. Dogra plantea una realineación endoscópica mediante abordaje retrógrado y anterógrado simultáneo, manteniendo la sonda uretral posteriormente durante 6 semanas en pacientes con lesiones uretrales traumáticas; las pacientes deben seguir autocateterismos tras la retirada de la misma; por tanto consideran como criterio indispensable la motivación por parte de las pacientes para realizarlos ${ }^{21}$.

\section{Tratamiento de las estenosis recidivadas en la mujer}

Si la estenosis uretral femenina es ya una patología poco frecuente, aún lo es más la estenosis recidivada, constituyendo hoy en día un desafío terapéutico para el urólogo. No existe ninguna técnica de elección con una eficacia claramente demostrada a largo plazo. Lo que sí parece demostrado es que aquellas pacientes que presenten defectos parciales o totales de uretra o fracasos repetidos de técnicas menos invasivas como la dilatación o la uretrotomía serán tributarias de técnicas de reconstrucción uretral $^{10}$. El mejor tratamiento quirúrgico en estos casos continúa siendo un asunto a debatir, sin existir un consenso claro de cuál es la mejor opción quirúrgica en estos casos.

Se realizó una revisión bibliográfica en la base de datos de Medline (PubMed) entre julio y diciembre de 2008, de los artículos publicados sobre el tratamiento de la estenosis uretral en la mujer entre 1995 y 2008. En Medline la búsqueda se siguió utilizando los siguientes términos: "female urethral estenosis", "female urethral reconstruction" y "female urethral estenosis treatment". Se revisaron los artículos publicados en inglés y castellano, y se descartaron revisiones sobre patología malformativa y pediátrica.

Palou et al. ${ }^{22}$ describen la primera técnica hallada en nuestra revisión mediante el uso de un colgajo de vagina en una única paciente con estenosis medio-distal yatrógena postcoagulación de condilomas. Describen la uretroplastia mediante el uso de 
colgajo de pared vaginal anterior aproximándolo a la cara posterior uretral previamente incidida $\mathrm{y}$ cubriéndola con los extremos de la pared vaginal que quedan a ambos lados de forma transversal. Dejan una sonda Foley 20Ch durante 3 días y cistostomía durante 3 semanas. El control se realiza con flujometría a los 10 meses, siendo ésta normal.

En 2002 Tanello $^{10}$ publica la uretroplastia con pedículo de labio menor en 2 pacientes con estenosis uretral fallida a tratamientos previos. La razón por la que se escogió la piel de labio menor fue su elasticidad, falta de vello y ser un tejido húmedo de fácil obtención. Se incinde la pared vaginal anterior desde el meato, separando la pared de la uretra, continuando la disección de forma circular por el tejido conectivo periuretral. Seccionan el grosor de la uretra ventralmente desde el meato hasta tejido sano proximal a la estenosis. El flap de labio menor se moviliza a partir del pedículo vascular y se aproxima a la uretra por transposición a través de un túnel por debajo de la pared vaginal. La cara epidérmica del flap se colocó sobre la luz uretral y se sutura a los márgenes mediante sutura continua extendiéndolo sobre sonda fenestrada que se retira a las 2 semanas previa uretrografia. El seguimiento presentado es de 24 meses con cistouretrografia normal y ausencia de residuo potmiccional pero sin utilizar parámetros urodinámicos para descartar la obstrucción. Estos autores concluyen que la reconstrucción uretral pasa por una correcta exposición del lecho uretral, una buena movilización del flap para evitar tracción sobre las suturas para lo que se requiere dejar un buen pedículo vascular. Y abogan por que si existe insuficiencia esfinteriana, concomitantemente colocar sling pubovaginal.

Montorsi $^{5}$ en el mismo año publica una serie de 17 pacientes a los que trataron mediante uretroplastia con flap vestibular evaluándolos no sólo anatómicamente sino también funcionalmente con parámetros urodinámicos. Los pacientes presentaban estenosis obstructivas de tercio distal y meato uretral y a todas las pacientes se les pasó un cuestionario de síntomas de la AUA. La técnica se siguió bajo microcirugía utilizando lentes de aumento $(4,5 \mathrm{x})$, realizándose una incisión perimeática en $\mathrm{Y}$ invertida, disecando los $3 \mathrm{~cm}$ distales de uretra entre las 3 y 9 horas. Se incide la uretra por su cara dorsal y se seccionó el fragmento estenótico que se remitió a anatomía Patológica. Se seccionó un pedículo de vestíbulo vaginal de $1 \times 3 \mathrm{~cm}$ inmediatamente supe- rior al meato, suturando su extremo distal al extremo distal de la uretra ( próximo al meato), uniendo los márgenes del pedículo a los uretrales para conformar el techo de la neouretra. Se retira la sonda vesical a las $24 \mathrm{~h}$ postcirugía y al mes se evalúan evidenciando mejoras en el 88\% de los pacientes en todos los parámetros analizados: Cuestionarios, Flujo máximo, Pdet en flujo máximo, Residuo postmiccional y cistouretrografia.

Schwender ${ }^{23}$ publica la primera serie de 2 instituciones en el tratamiento de la estenosis mediante uretroplastia ventral con flap vaginal. Presentan una serie de 8 pacientes, en los que se realiza una incisión sobre pared vaginal anterior en U invertida con el vértice debajo del meato uretral. Se diseca el plano entre la pared vaginal y la fascia periuretral hasta conseguir un flap de $3 \mathrm{~cm}$. Se introduce un espéculo nasal y se incide la zona estenótica a las $6 \mathrm{~h}$. Se sutura el flap de vagina sobre el defecto invirtiéndolo sobre sí mismo, de manera que el vértice de la $U$ se sutura al extremo de la incisión sobre la uretra, suturando los márgenes de la vagina a los de la mucosa uretral. Se comprueba el paso de un cistoscopio $22 \mathrm{Ch}$ y se deja sonda Foley durante 7-10 días. Estos pacientes presentan una mejoría en el calibre uretral y disminución de los residuos postmiccionales y, con un seguimiento de 2,5 años sólo una paciente ha requerido dilataciones posteriormente. Los autores defienden esta técnica por considerarla de fácil realización y reproducción, no requiere tunelizar tejido y aseguran que se tolera mejor que la incisión suprameática. No presentan resultados urodinámicos y tampoco queda claro cuales son los criterios diagnósticos que utilizan.

Un año antes Park presenta una serie de 7 pacientes pediátricos tratados mediante uretroplastias con injerto de mucosa bucal, sólo en una de las pacientes la patología de la uretra fue la estenosis yatrógena con fístula uretrovaginal añadida (siendo el resto pacientes pediátricas con malformaciones uroginecológicas) ${ }^{24}$. En este caso, se escindió la uretra fibrótica hasta encontrar bordes sanos. Para realizar el injerto se secciona un rectángulo de $2 \mathrm{x} 4 \mathrm{~cm}$ de mucosa por debajo del conducto Stenon, se sutura los bordes del injerto y se tubuliza in situ alrededor de una sonda Foley 8-10 Ch. Se secciona parte de mucosa vaginal sobre la uretra dañada para permitir llevar un flap glúteo cuya sutura no coincida con el injerto. Un flap vascularizado de glúteo o labio suficientemente largo para alcanzar el 
esfinter se usó para cubrir la neouretra, cuidando de dejar la base amplia y gruesa para asegurar la vascularización. Se mantuvo la sonda vesical durante 2 semanas. En el control postoperatorio a los 12 meses de seguimiento la paciente presenta micciones satisfactorias por uretra pero presentó como complicación la recidiva de la fístula uretral. Estos autores concluyen que existe cierta dificultad para el uso de injertos con tejidos libres para la reconstrucción uretral femenina por falta de tejido local que permita una buena vascularización, solventando dicho inconveniente con la utilización de flaps de glúteo y periné.

Berglund $^{25}$ en 2006 es el primero en publicar la uretroplastia ventral con mucosa bucal en 2 pacientes a partir de la experiencia de Park. El autor recomienda esta técnica cuando no sea posible utilizar la pared vaginal para la realización de un flap. El diagnóstico en estas pacientes se realizó mediante estudios de Presión/Flujo y uretrocistoscopia. La uretra se expuso a través de una incisión en la línea media de la pared anterior vaginal, abriendo la uretra a las $6 \mathrm{~h}$ desde el meato hasta tejido sano, suturando el injerto sobre éste defecto y se cubre con tejido sano periuretral o un flap de Martius. Se deja tutorizado con S. Silastic $18 \mathrm{~F}$ y catéter suprapúbico $22 \mathrm{~F}$. Se realiza control a las 6 semanas mediante cistoscopia y cistouretrografia. Una de las pacientes desarrolló estenosis de meato que requirió dilataciones posteriormente, ninguna de las pacientes presentó incontinencia de esfuerzo posterior. Los autores reportan que las pacientes se encuentran asintomáticas y con exploraciones normales a los 30 y 12 meses de seguimiento. No presentan parámetros urodinámicos de control.

Migliari $^{26}$ el mismo año publica un procedimiento similar en 3 pacientes, pero el injerto se colocó en posición dorsal. Realizan el diagnóstico mediante estudios de Presión-lujo y uretrocistoscopia. Una de las pacientes tenía una uretroplastia previa. Se realiza una incisión en $U$ invertida sobre meato de 3-9 h, y se crea un plano dorsal entre el cuerpo del clítoris por encima y la uretra, por debajo, lo más cerca posible de la uretra para evitar dañar al clítoris e identificando el esfinter urinario. Se realiza incisión dorsal en todo el grosor uretral desde el meato hasta el cuello vesical y sobre el defecto se sutura un injerto de mucosa bucal de 3x5 $\mathrm{cm}$. Se retira el catéter uretral a los 15 días. Con 6 meses de seguimiento medio los pacientes presentan flujometría normal y un nomograma Blaivas-
Groutz no obstructivo, ninguna de las pacientes ha presentado incontinencia. Las ventajas de ésta técnica son, según los autores, que la uretroplastia con colgajo bucal dorsal en mujeres ofrece mayor soporte mecánico lo que disminuye el riesgo de saculaciones y neovascularización; Permite una reconstrucción uretral más fisiológica posibilitando modelar el meato uretral hacia arriba y así conseguir una micción más fisiológica. Además mantiene intacta la cara ventral de la uretra para eventuales intervenciones antiincontinencia en uretra media.

\section{CONCLUSIONES}

La estenosis uretral en la mujer como causa de obstrucción infravesical es una entidad poco frecuente sin un diagnóstico claro plenamente establecido. Parece lógico pensar que serán etiquetadas de estenosis de uretra aquellas pacientes con diagnóstico urodinámico de obstrucción que presenten melladuras o muescas en las pruebas de imagen, imposibilidad para el sondaje o se evidencie una imagen de estrechez en la uretroscopia. Dichas pacientes serán tributarias de tratamiento desobstructivo.

El tratamiento mediante dilataciones de los síndromes uretrales sin causa claramente obstructiva debe evitarse, ya que no existe evidencia científica sobre el beneficio de las dilataciones uretrales para mejorar la sintomatología del tramo urinario inferior en la mujer.

Ante una estenosis de uretra tendremos que valorar preoperatoriamente la longitud, localización con respecto al esfínter urinario y la integridad de éste, el estado del resto de uretra indemne y si la paciente ha recibido tratamientos previamente.

Los algoritmos terapéuticos muestran el manejo inicial para las estenosis cortas en la mujer mediante dilataciones y/o uretrotomia interna, aunque debemos advertir a la paciente la posibilidad de recidiva y valorar su destreza manual de cara a realizar autocateterismos.

En los casos de estenosis recidivadas o largas debe plantearse la uretroplastia mediante flap vaginal con un pedículo ancho que asegure una buena vascularización y interponiendo tejido periuretral o un flap de Martius. Otra opción seria la uretroplastia con mucosa bucal en el que el abordaje posterior sería la opción a considerar. Teniendo en cuenta lo extraordinario de estos casos se debería considerar referir a estas pacientes a centros especializados. 


\section{REFERENCIAS}

1. Groutz A, Blaivas JG and Chaikin DC: Bladder outlet obstruction in women: definition and characteristics. Neurourol Urodyn. 2000;19(3):213-220.

2. Podesta ML, Jordan GH. Pelvic fracture urethral injuries in girls. J Urol. 2001;165(5):1660-1665.

3. Noe NH. Long-term follow-up of endoscopic management of urethral strictures in children. J Urol. 1987;137(5):951-953.

4. Karam I, Droupy S, Abd-Alsamad I, Uhl JF, Benoît G, Delmas V. Innervation of the Female Human Urethral Sphincter: 3D Reconstruction of Immunohistochemical Studies in the Fetus. Eur Urol. 2005 May;47(5):627-633;discussion 634.

5. Montorsi F, Salonia A, Centemero A, Guazzoni G, Nava L, Da Pozzo LF et al. Vestibular Flap Urethroplasty for Strictures of the Female Urethra. Urol Int. 2002; 69(1):12-16.

6. Thind P. The Significance of Smooth and Striated Muscles in the Sphincter Function of the Urethra in Healthy Women. Neurourol Urodyn. 1995;14(6):585-618.

7. Arango Toro O, Doménech Mateu JM. Evidencias anatómicas y clínicas sobre el pudendo intrapélvico y su relación con el esfinter estriado de la uretra. Actas Urol Esp. 2000;24(3):248254.

8. Stein TA, Delancey JO. Structure of the perineal membrane in females: gross and microscopic anatomy. Obstet and Gynecol. 2008; $111(3): 686-693$.

9. Keegan KA, Nanigian DK, Stone AR. Female urethral stricture disease. Curr Urol Rep. 2008;9(5):419-423.

10. Tanello M, Frego E, Simeone C, Cosciani Cunico S. Use of Pedicle Flap from the Labia Minora for the Repair of Female Urethral Strictures. Urol Int. 2002;69(2):95-98.

11. Romero Pérez P, Schiefenbusch Munne E, Lobato Encinas JJ, Mira Llinares A. Estenosis uretral femenina por distrofia vulvar. Arch Esp Urol. 1990;43(4):341-346.

12. Smith AL, Ferlise VJ, Rovner ES. Female urethral strictures: successful management with long-term clean intermittent catheterization after urethral dilatation. BJU International. 2006; 98(1):96-99.

13. Edwards LE, Lock R, Powell C, Jones P. Post-catheterisation urethral strictures. A clinical and experimental study. $\mathrm{Br} J$ Urol. 1983;55(1):53-56.

14. Goldman HB, Zimmern PE. The treatment of female bladder outlet obstruction. BJU Int. 2006 Sep;98 Suppl 1:17-23; discussion 24-26.
15. Brannan D. Stricture of the female urethra. J Urol. 1951;66(2): 242-253.

16. Pugh WS. Stricture of the female urethra. Ann Surg. 1924 May; 79(5):770-778.

17. Lyon RP, Smith DR. Distal uretrhal stenosis. J. Urol. 1963;89 (3):414-421.

18. Takao M, Asano T, Nakamura H. Urodinamic studies before and after urethral dilatation with metal sounds for female urethral stricture. Hinyokika Kiyo 1992;38(1):15-17.

19. Noe HN. Long-term followup of endoscopic management of urethral strictures in children. J Urol. 1987 May;137(5):951953.

20. Lemack GE, Foster B, Zimmern PE. Urethral dilation in women: a questionnaire-based analysis of practice patterns. Urol 1999;54(1):37-43.

21. Dogra PN, Nabi G. Endourologic reconstruction of post-traumatic obliterated urethral stricture in a young female: a point of technique. Urology. 2001 Dec;58(6):1053-1055

22. Palou J, Caparros J, Vicente J. Use of proximal- based vaginal flap in stricture of the female urethra. Urology. 1996;47(5):747749 .

23. Schwender CE, Ng L, McGuire E, Gormley EA. Technique and results of urethroplasty for female urethral strictures. J Urol. 2006 Mar;175(3 Pt 1):976-980; discussion 980.

24. Park JM, Hendren WH. Construction of female urethra using buccal mucosa graft. J Urol. 2001 Aug;166(2):640-643.

25. Berglund RK, Vasavada S, Angermeier K, Rackley R. Buccal mucosa graft urethroplasty for recurrent stricture of female urethra. Urol 2006, 67(5):1069-1071.

26. Migliari R, Leone P, Berdondini E, De Angelis M, Barbagli G, Palminteri E. Dorsal Buccal Mucosa Graft Urethroplasty for Female Urethral Strictures. J Urol. 2006 Oct;176(4 Pt 1):14731476.

Correspondencia autor: Dra. Cristina Gutiérrez Ruiz Servicio de Urología. Fundación Puigvert

Cartagena, 340-350 - 08025 Barcelona

Tel.: 934169700

E-mail autor: cgutiruiz@yahoo.es / cgutierrez@fundacio-puigvert.es Información artículo: Original - Estenosis uretral

Trabajo recibido: septiembre 2008

Trabajo aceptado: abril 2009 